\title{
EVALUACIÓN DEL MECANISMO DE PAGO CAPITADO EN EL SEGURO INTEGRAL DE SALUD DEL PERÚ
}

\author{
ASSESSMENT OF THE MECHANISM OF PAYMENT CAPITATED IN THE \\ PERUVIAN SEGURO INTEGRAL DE SALUD \\ Pedro Grillo-Rojas ${ }^{1, a}$, Ana Vásquez ${ }^{2, b}$
}

\begin{abstract}
RESUMEN
Objetivo: Evaluar el impacto del mecanismo de pago capitado, implementado por el Seguro Integral de Salud (SIS), en la ejecución de los recursos provenientes de la fuente de financiamiento donaciones y transferencias, durante los años 2012 al 2016, Perú. Métodos: Se evaluó los porcentajes de ejecución de las regiones, el número de prestaciones preventivas versus el número de prestaciones recuperativas y se midió el impacto en el incremento de las atenciones preventivas en las regiones piloto versus las regiones del contrafactual. Se realizó un estudio descriptivo retrospectivo pre post y diferencia de diferencias entre las regiones piloto Apurímac, Amazonas, Ayacucho, Callao y Huancavelica comparados con un contrafactual apareado por Índice de Desarrollo Humano; además, se consideró a las regiones de Moquegua, Loreto, Puno, Cajamarca y Huánuco. Resultados: Se observó un incremento del porcentaje promedio de ejecución, previo al mecanismo capitado, de $68,5 \%$ en el 2012 al 92,3\% el 2016; asimismo, el incremento a nivel nacional de las prestaciones preventivas versus las prestaciones recuperativas, llegando invertirse el peso específico de las prestaciones preventivas a favor de las mismas y mediante el método de diferencias en diferencias se evidenció que las regiones piloto tuvieron una diferencia positiva de 1551 prestaciones preventivas por cada 10000 asegurados sobre las regiones del contrafactual.
\end{abstract}

Palabras clave: Impacto; Mecanismo de Pago; Capitado; Pre Post; Resultados Sanitarios; Resultados Intermedios; Impacto; Diferencia en diferencias; Sistema de Aseguramiento Público. (fuente: DeCS BIREME)

\begin{abstract}
Objective: To Assess the impact of the capitated payment mechanism, implemented by the Comprehensive Health Insurance (SIS), in the execution of resources from the source of financing, donations and transfers, during the years 2012 to 2016, Peru. Methods: Assessment of the execution percentages of the regions, the number of preventive benefits versus the number of recuperative benefits and the impact on the increase of preventive care in the pilot regions versus the counterfactual regions was measured. A retrospective descriptive study was carried out pre post and difference of differences between the pilot regions Apurímac, Amazonas, Ayacucho, Callao and Huancavelica compared with a counterfactual paired by the Human Development Index; In addition, the regions of Moquegua, Loreto, Puno, Cajamarca and Huánuco were considered. Results: An increase in the average percentage of execution was observed, prior to the capitated mechanism, from $68,5 \%$ in 2012 to $92,3 \%$ in 2016; likewise, the increase at the national level of preventive benefits versus recuperative benefits, with the specific weight of preventive benefits being invested in favor of them and, using the difference in differences method, it was evident that the pilot regions had a positive difference of 1551 preventive benefits for every 10,000 insured persons versus the counterfactual regions.
\end{abstract}

Key words: Impact; Payment Mechanism; Capitated; Pre Post; Sanitary Results; Intermediate results; Capitated; Payment mechanisms; Impact; Difference in differences; Health results; Intermediate results; Public insurance system. (source: MeSH NLM)

\footnotetext{
${ }^{1}$ Facultad de Medicina Humana - UPSMP, Lima-Perú.

${ }^{2}$ Facultad de Salud Pública y Administración - UPCH, Lima-Perú.

a Médico especialista en administración.

Médico especialista en administración, especialidad de salud pública con mención en epidemiología.
}

Citar como: Pedro Grillo-Rojas, Ana Vásquez. Evaluación del mecanismo de pago capitado en el seguro integral de salud del Perú. Rev. Fac. Med. Hum. Julio 2019; 19(3):11-18. DOI 10.25176/RFMH.v19i3.2168 


\section{INTRODUCCIÓN}

Uno de los principales problemas que ha enfrentado el Sistema de Salud en el Perú, es el uso eficiente de los recursos destinados al funcionamiento de los establecimientos de salud en los distintos niveles de gobierno; ya sean los gestionados por el Ministerio de Salud, EsSalud, otros ministerios o los gobiernos regionales. No obstante, esta situación a partir del 2015, el Acuerdo Nacional del Perú reconoce el acceso universal a los servicios de salud y a la seguridad social como una política de Estado'.

Esta política de Estado conllevó durante estos años a un incremento en el presupuesto del sector salud y el acceso de mayor número de peruanos a los servicios de salud a través del Seguro Integral de Salud (SIS). Este incremento progresivo, entre los años 2013 - 2015, significó un paso importante en el afianzamiento del acceso a la salud, pero a su vez, supuso una serie de retos que debían afrontar los gestores, tanto desde el ámbito de la financiadora pública SIS como desde los prestadores de servicios de salud, por lo que era necesario reevaluar las formas de relación desarrolladas desde el año 2002 hacia adelante.

Desde entonces en el Perú, se vienen implementando estrategias dirigidas a definir un modelo de financiamiento público en salud basado en impuestos, debido al lento crecimiento de la formalidad que serviría de fuente de financiamiento para un modelo Bismarckiano en el ámbito de la seguridad social peruana.

El año 2009, se promulga la Ley 29344, Ley Marco del Aseguramiento Universal en Salud y su Reglamento, donde se establece los roles de financiación para las Instituciones Administradoras de Fondos de Salud (IAFAS) públicas y privadas; así como, el rol prestador para las Instituciones Prestadoras de Servicios de Salud (IPRESS) de carácter público o privado. La mencionada ley, define al SIS como una IAFAS, bajo el principio de irreversibilidad²; es decir, que se encuentra fuera del ámbito de los programas sociales. En ese marco, se inició una serie de acciones dirigidas a establecer un vínculo de tipo contractual o acuerdo entre el SIS y las IPRESS públicas ${ }^{3}$.

Las relaciones entre IAFAS e IPRESS se encuentran fuertemente relacionadas al mecanismo de pago y la forma en que este se retribuye a las IPRESS. Por ello, la forma en que los recursos recaudados, en un Sistema de Salud, se convierten en servicios para las personas es un elemento crítico en la ecuación entre salud y dinero. "Esta es la denominada función de compra, que consiste en hacer que los recursos recaudados de cualquiera de las fuentes mencionadas se conviertan en servicios de la manera más eficiente posible" 4 .

Se sabe que existen diferentes tipos de mecanismos de pago en la relación entre financiadores y prestadores de salud. Al respecto, el presente estudio se va a centrar en analizar el mecanismo de pago capitado, consistente en transferencias financieras realizada por el SIS, a los establecimientos de salud en forma anticipada, tomando como cálculo un monto por cada asegurado. Dicho mecanismo "ha demostrado desincentivar el exceso de servicios que si se observa en el caso del pago fee for service" 5 .

El mecanismo de pago se inició como piloto en la Región Huancavelica el 2011; al año siguiente se replicó a las Regiones de Amazonas, Ayacucho, Apurímac y Callao. En ese mismo ejercicio fiscal, se emitió la Resolución Jefatural 149-2012-SIS6, en la cual se establece el procedimiento de cálculo de transferencias desde el SIS hacia las IPRESS públicas, bajo la modalidad capitado prospectivo y, para el año 2013 el capitado se extendió hacia todo el país. Posteriormente, en el 2016, se modifican algunos procedimientos del cápita mediante la Resolución Jefatural 009-2016/SIS7 , ya que el SIS sólo transfería presupuestos.

En las relaciones principal agente, que marcan la forma en que interaccionan las IPRESS públicas con la IAFAS públicas -SIS, se eligió el mecanismo de pago basado en la capitación en un marco de presupuesto cerrado, que sólo significaba alrededor del $6 \%$ del presupuesto público asignado a la función salud del sector $y$ gobiernos regionales ${ }^{8}$. El pago capitado del Seguro Integral de Salud ejercía un mecanismo de distribución y contención de costos al apostar por la prevención y compartir el riesgo con las IPRESS, los cuales estaban dirigidos a conseguir, en la cadena de causalidad, logros a nivel de resultados intermedios. Definidos en tres objetivos:

$1^{\circ}$ Previsibilidad del valor a transferir a las IPRESS Públicas, lo cual le permitía al prestador planificar de manera más real las compras de productos farmacéuticos, productos sanitarios y dispositivos médicos.

$2^{\circ}$ Incrementar las atenciones preventivas versus las atenciones recuperativas, puesto que el incentivo para ejecutar las prestaciones no dependían del consumo de productos farmacéuticos o dispositivos médicos; sino que se incentivaba a brindar el paquete preventivo para cada etapa de vida.

$3^{\circ}$ Incrementar la ejecución de los recursos transferidos 
a las regiones por Donaciones y Transferencias a través del SIS; así como el presupuesto asignado y ejecutado en el primer nivel de atención versus el segundo y el tercer nivel de atención.

Estos objetivos, a diferencia de los pagos capitados tradicionales en el mercado privado de aseguramiento en salud, no fueron diseñados únicamente para transferir riesgo sino para modular el comportamiento de las IPRESS.

El SIS define el mecanismo de pago capitado como un tipo de contrato que crea incentivos específicos en relación a la prestación médica. Estos contratos son herramientas importantes para los gestores de salud, pues hay evidencia de que modifican la conducta de otro agente alterando la productividad, calidad y composición de las prestaciones, la distribución de la oferta, la previsibilidad en relación al gasto del prestador, entre otros.

El pago capitado es prospectivo y se realiza al primer nivel de atención. El poder del modelamiento mencionado radica en dos componentes financieros: el fijo, condicionado sólo a la firma del convenio y que garantiza las prestaciones y; el variable, condicionado al cumplimiento de indicadores prestacionales en el marco de Presupuesto por Resultados. Adicionalmente, se busca modificar conductas prestacionales, fomentando la actividad preventiva por sobre la recuperativa $y$ fortaleciendo el primer nivel de atención. Finalmente, se esperan mejoras en los resultados sanitarios y en la Salud Pública.

Por lo tanto, el SIS no planteó indicadores dirigidos a mejorar la calidad del servicio ni de resultados finales como la disminución de la mortalidad materna o anemia, puesto que dichos objetivos dependían de otras fuentes de financiamiento, principalmente de los Recursos Ordinarios que transfería el MEF al Ministerio de Salud y a los gobiernos regionales; de los Recursos Directamente Recaudados, incluso de la intervención de otros sectores y de otras externalidades que no eran controladas a través del Seguro Integral de Salud. Sin embargo, no sólo era necesario lograr objetivos ligados a transferencias, sino incentivar la eficiencia en la distribución de dichas transferencias. Además, relacionarlas al cumplimento de los objetivos sanitarios nacionales, por cuanto el presupuesto del Seguro Integral de Salud experimentó un incremento muy importante durante los años 2013 al 2015

Entre las dificultades encontradas, tenemos:

1. Procedimientos engorrosos en las unidades ejecutoras, que no necesariamente facilitaban que los recursos, asignados por el SIS de manera prospectiva, lleguen directamente a los establecimientos de salud.

2. La falta de recursos humanos suficientes que puedan llegar a la población dispersa.

3. La falta de compras planificadas para el logro de la meta planteada. En esta lógica, se fue incluyendo otras variables, como la distribución de los medicamentos e insumos hasta el establecimiento de salud y no únicamente al almacén regional.

4. La falta de capacidades en gestión, por lo que fue necesaria la incorporación de los comités de gestión, que autorizaran los gastos por los acuerdo tomados a partir de los establecimientos de salud de una unidad ejecutora.

5. La necesidad de incorporación de figuras de control financiero a través de la obligatoriedad de llenado de los registro de Sistema Integrado de Gestión Administrativa (SIGA), además del Sistema de Aseguramiento del SIS (SIASIS) para poder realizar el monitoreo del gasto.

\section{METODOLOGÍA DE CÁLCULO DEL MECANISMO CAPITADO:}

Para el cálculo del mecanismo capitado, desde el 2012 hasta el 2015, se utilizó dos vertientes de cálculo:

i) Por estimación histórica y proyección para la asignación del presupuesto por las prestaciones recuperativas (atenciones y atendidos) y la intensidad de uso promedio histórica, $\mathrm{y}$

ii) Por estimación paramétrica para los servicios preventivos en base al número de prestaciones que deberían realizarse para cumplir con lo establecido en la normatividad vigente del Ministerio de Salud.

Este último parámetro era influenciado por dos indicadores extensión de uso e intensidad de uso, lo cual formaba parte de la negociación con cada región.

Al respecto, el 2007, se debe mencionar que se encontró literatura chilena donde se analiza un modelo de cálculo similar, basado en el cálculo de prestaciones programables y las prestaciones ligadas a morbilidad o denominadas espontáneas?.

Otra peculiaridad de la metodología es que el cálculo de la transferencia, denominada cápita, se desarrollaba por cada región, no era un cálculo nacional. Se consideró la realidad de cada región y se modeló escenarios de incremento de afiliación, entre otros ${ }^{10}$.

Una vez calculadas las proyecciones de las prestaciones de salud, se procedía a la valorización de las mismas de 
acuerdo a la tarifa SIS. Esta tarifa no significaba el costo total de la prestación; por cuanto, como hemos definido anteriormente, la asignación de recursos a través de la fuente SIS (Donaciones y Transferencias) cubría sólo cerca del $7 \%$ del presupuesto público para la función salud.

$\mathrm{P}=$ Prestaciones de salud proyectadas.

$\mathrm{Q}=$ Costo unitario por prestación a tarifa SIS.

Posteriormente, el valor resultante de $\left(P^{*} Q\right)$ era sometido a ajustes por indicadores; por ejemplo, el incremento de la afiliación durante el año siguiente, la extensión de uso en prestaciones preventivas, el porcentaje de asegurados con paquetes completos en prestaciones preventivas y otros ajustadores de riesgo como: ruralidad, índice de pobreza y migración que se negociaban con el prestador de servicios de salud. Luego, el resultado de dicha ecuación se dividía entre el número de asegurados de la región con lo cual se establecía el valor per cápita. Finalmente, desde el 2016 el mecanismo fue modificado sustancialmente y eliminado hacia el año 2018.

Costo total / Número de asegurados = Costo per cápita

Se implementaron indicadores prestacionales y financieros ajustados, progresivamente, los siguientes años. Las características principales de estos indicadores se encontraban relacionados a la salud reproductiva y gestación, a niños y a enfermedades priorizadas no transmisibles y transmisibles.

Es por esto que el objeto de este estudio es realizar una evaluación del mecanismo de pago denominado cápita, desarrollado en el Seguro Integral de Salud entre los años 2012 al año 2016.

\section{MÉTODOS}

Se realizó una evaluación descriptiva del cumplimiento de los objetivos de la implementación del mecanismo de pago denominado "cápita del Seguro Integral de Salud" a nivel I, para el porcentaje de ejecución por la fuente de financiamiento Donaciones y Transferencias a nivel de actividades y la evolución del número de prestaciones preventivas y recuperativas en el transcurso de los años previos y durante la implementación del mecanismo evaluado.

Asimismo, una evaluación cuasi experimental para comparar el incremento de las prestaciones preventivas durante el periodo del piloto del mecanismo de pago capitado entre las regiones incluidas versus las cuales no fueron incluidas tomando como base las prestaciones del año 2011 y comparándolas con las del año 2012.

Se realizó un análisis secundario de la ejecución presupuestal de los gobiernos regionales de Apurímac, Amazonas, Ayacucho, Callao, Huancavelica, Moquegua, Loreto, Puno, Cajamarca y Huánuco en lo concerniente a las fuentes de financiamiento Donaciones $y$ Transferencias y Recursos Ordinarios, periodo 2009 2016, de las siguientes fuentes de información:

1. Consulta amigable del MEF.

2. Data ingresada al SIASIS por los prestadores de servicios de salud a nivel nacional.

3. Data de número de asegurados al $\mathrm{SIS}^{11}$.

El método para la evaluación del porcentaje de ejecución de los recursos transferidos por el SIS a las regiones será el siguiente:

La información de la "Consulta Amigable" del MEF será analizada para el periodo 2009 - 2016, comparando los niveles de ejecución de los años previos a la implementación del cápita y los años de implementación a nivel de los gobiernos regionales, para los recursos transferidos en la función salud por la fuente de financiamiento "Donaciones y Transferencias", sólo actividades, y se comparará con los recursos asignados por "Recursos Ordinarios" para los mismos años, bajo los mismos criterios.

Para la comparación entre regiones cápita y no cápita entre el 2011 y 2012 se utilizó la información del SIASIS y para evaluar el impacto se utilizó la metodología denominada "Diferencia de Diferencias". Además las regiones que formaron parte del contrafactual estuvieron apareadas por el Índice de Desarrollo Humano con las Regiones que se encuentran en el grupo de intervención.

Con el fin de homologar los resultados, no se utilizó el número absoluto de prestaciones sino la tasa de prestaciones por diez mil asegurados SIS.

La fórmula empleada para cada comparación fue la siguiente:

$X=\left(Y_{t 1}-Y_{T 0}\right)-\left(Y_{C 1}-Y_{C 0}\right.$

Donde:

$X=$ Impacto en relación a incremento de número de prestaciones preventivas

$\mathrm{Y}_{\mathrm{t} 1}-\mathrm{Y}_{\mathrm{T} 0}$ : Número de prestaciones al final y al inicio en las regiones de la intervención.

$Y_{c 1}-Y_{c 0}$ : Número de prestaciones al final y al inicio en 
las regiones en las que no se desarrolló la intervención.

\section{RESULTADOS}

\section{Porcentaje de ejecución:}

La Figura 1, muestra que, a partir del 2013, se observa un

incremento importante en la ejecución de los recursos transferidos hasta llegar al 92,36\% de ejecución 2015.

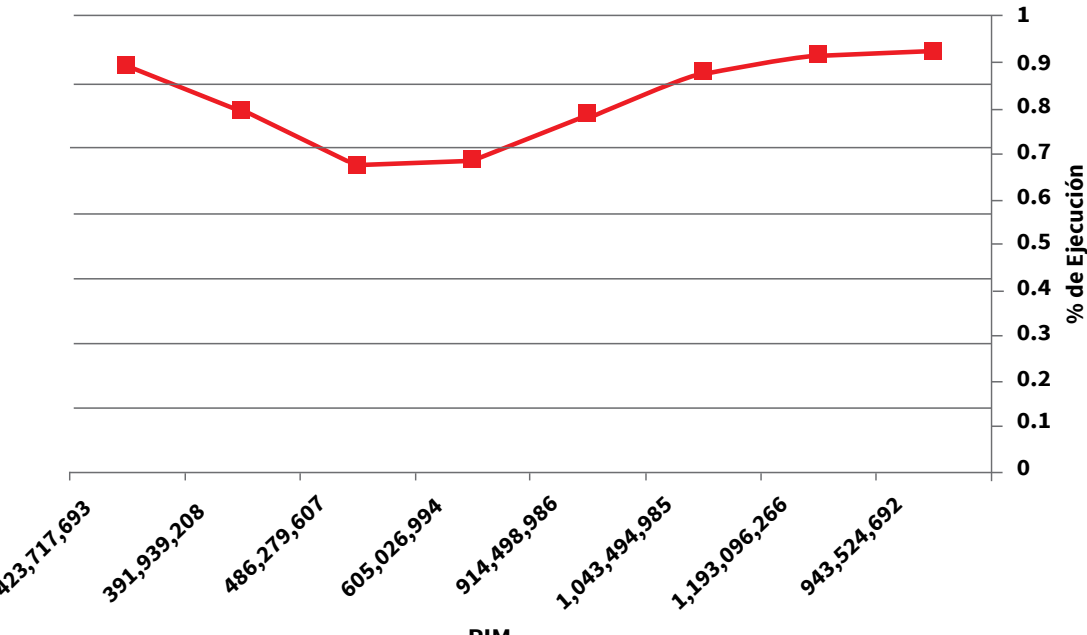

$r=0.54$

Fuente: Consulta Amigable MEF.

Figura 1. \% de ejecución por fuente donaciones y transferencias a nivel regional 2009 - 2016.

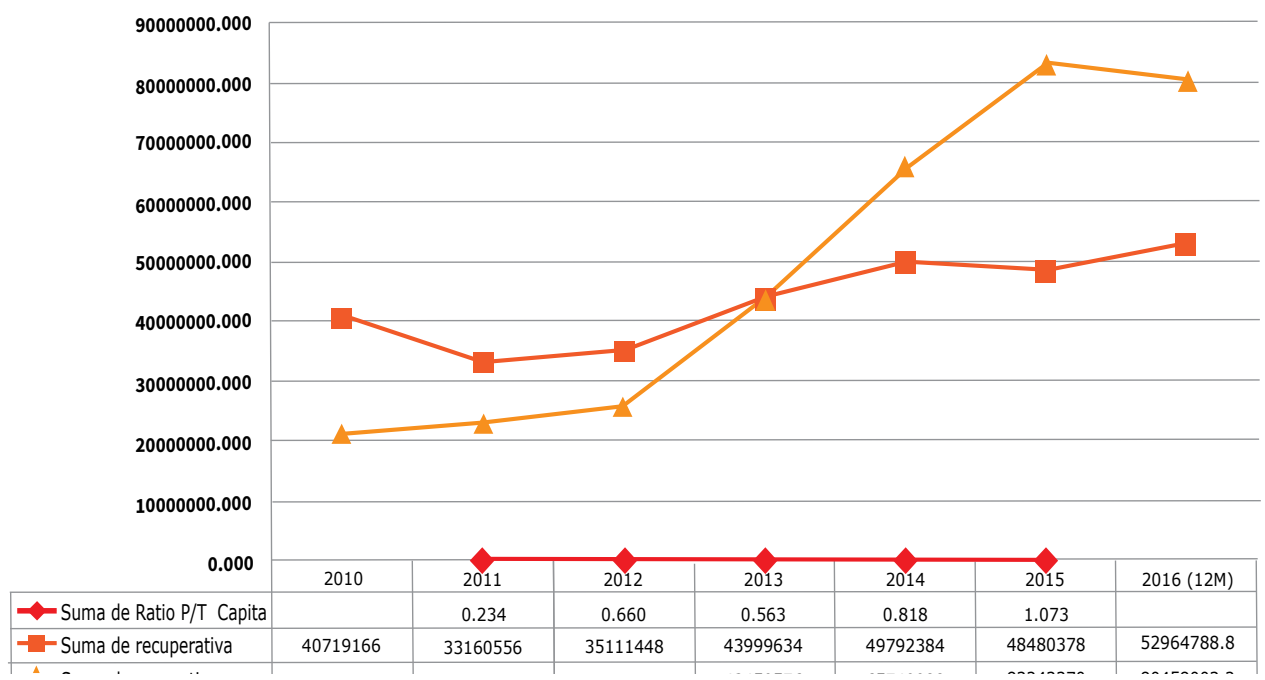

Fuente: Presentation: Dr. Jose del Carmen Sara Innovations in Financing and Payment for UHC - PERÚ - Joint Learning Network for Universal Health Coverage Learning Exchange on Financing and Payment Models for Primary Health Care - In-person meeting Santiago, Chile August 1-4, 2016 - Results of the Innovation.

Figura 2. Incremento de las prestaciones preventivas y recuperativas.

Al hacer la evaluación del crecimiento de las prestaciones desde el año 2010 al 2016, se observó que partir del año 2013 se genera una intersección entre las líneas de atenciones recuperativas y las atenciones preventiva; esto demuestra, por primera vez, la inversión en relación a las prestaciones preventivas versus las recuperativas financiadas por el SIS. Además, dicho incremento llegó a ser cuatro veces mas que el número de prestaciones preventivas ingresadas en el SIASIS en el año 2012 (Figura 2). 


\section{Comparación grupo de regiones de intervención vs contrafactual:}

Las regiones del contrafactual fueron apareadas por año 2012, con las regiones que estuvieron bajo el Índice de Desarrollo Humano calculado para el la intervención (Tablas 1,2).

Tabla 1. Grupo de intervención.

\begin{tabular}{|cccccccc}
$\begin{array}{c}\text { Ubigeo } \\
\mathbf{2 0 1 0}\end{array}$ & Región & Población & $\begin{array}{c}\text { Índice de } \\
\text { desarrollo } \\
\text { humano }\end{array}$ & $\begin{array}{c}\text { Esperanza } \\
\text { de vida al } \\
\text { nacer }\end{array}$ & $\begin{array}{c}\text { Población } \\
\text { con } \\
\text { educación } \\
\text { secundaria } \\
\text { completa }\end{array}$ & $\begin{array}{c}\text { Años de } \\
\text { educación } \\
\text { (población } \\
\text { de 25 y } \\
\text { más) }\end{array}$ & $\begin{array}{c}\text { Ingreso } \\
\text { familiar } \\
\text { per cápita }\end{array}$ \\
\hline 070100 & Callao & 969,170 & 0,5863 & 79,16 & 81,01 & 10,35 & 822,6 \\
\hline 010000 & Amazonas & 417,508 & 0,3846 & 73,99 & 53,65 & 6,66 & 453,7 \\
\hline 030000 & Apurímac & 451,881 & 0,3444 & 72,41 & 60,57 & 6,15 & 330,8 \\
\hline 050000 & Ayacucho & 666,029 & 0,3336 & 70,22 & 43,59 & 6,38 & 358,7 \\
\hline 090000 & Huancavelica & 483,580 & 0,2962 & 65,16 & 43,16 & 5,58 & 317,2 \\
\hline
\end{tabular}

Tabla 2. Grupo contrafactual.

\begin{tabular}{|cccccccc} 
Ubigeo & Región & Población & $\begin{array}{c}\text { Índice de } \\
\text { desarrollo } \\
\text { humano }\end{array}$ & $\begin{array}{c}\text { Esperanza } \\
\text { de vida al } \\
\text { nacer }\end{array}$ & $\begin{array}{c}\text { Población } \\
\text { con } \\
\text { educación } \\
\text { secundaria } \\
\text { completa }\end{array}$ & $\begin{array}{c}\text { Años de } \\
\text { educación } \\
\text { (población } \\
\text { de 25 y } \\
\text { más) }\end{array}$ & $\begin{array}{c}\text { Ingreso } \\
\text { familiar } \\
\text { per cápita }\end{array}$ \\
\hline 180000 & Moquegua & 174,859 & 0,6215 & 77,76 & 80,74 & 9,64 & 1042,5 \\
\hline 160000 & Loreto & $1,006,953$ & 0,3977 & 70,49 & 43,61 & 8,09 & 500,1 \\
\hline 210000 & Puno & $1,377,122$ & 0,3942 & 67,52 & 74,04 & 7,49 & 426,3 \\
\hline 060000 & Cajamarca & $1,513,892$ & 0,3773 & 73,83 & 54,78 & 6,4 & 421,3 \\
100000 & Huánuco & 840,984 & 0,3746 & 72,33 & 45,47 & 6,73 & 448,4 \\
\hline
\end{tabular}

Fuente: Instituto Nacional de Estadística e Informática (INEI).

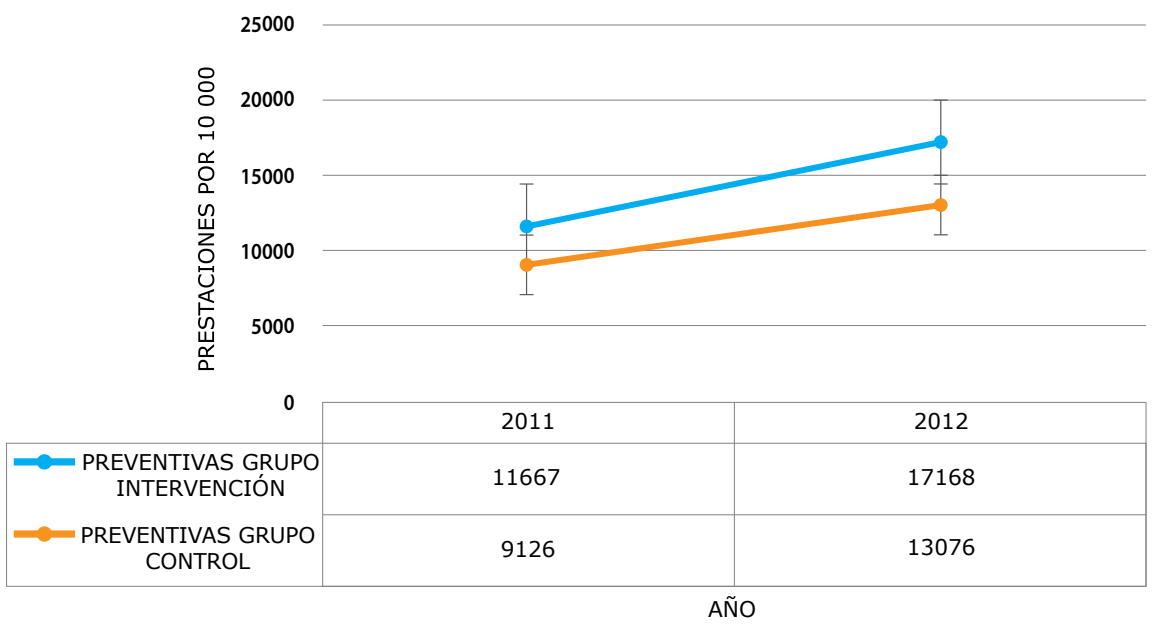

\section{$X=1551$ por 10000}

Fuente: SIASIS.

Figura 3. Atenciones preventivas grupo de intervención vs contrafactual.

El resultado de la comparación entre el grupo te intervención y el contrafactual arroja una diferencia positiva de 1551 atenciones preventivas más por cada 10000 asegurados del Seguro Integral de Salud a favor del grupo de regiones de la intervención durante el primer año en comparación con las atenciones preventivas del grupo contrafactual (Figura 3). 


\section{DISCUSIÓN}

Los resultados demuestran que el mecanismo de capitación desarrollado por el Seguro Integral de Salud generó un incremento significativo de la ejecución presupuestal total en la fuente "Donaciones y Transferencias" por parte de las regiones a nivel nacional, así como un incremento significativo de las actividades preventivas versus las recuperativas, estos resultados son concordantes con lo expresado en una reciente publicación ${ }^{12}$ en Ecuador, en la cual también se ha tomado en cuenta el factor positivo que tiene un mecanismo de pago por capitación en el incremento de las prestaciones preventivas.

Asimismo, aunque no en la misma medida concuerdan en parte con un artículo publicado en julio del $2018^{13}$ en el cual bajo una metodología similar a la utilizada en este estudio; se analizó resultados a nivel de enfermedad diarreica aguda, infección respiratoria en niños, anemia, muerte materna evitada en gestantes, cobertura de crecimiento y desarrollo y control del niño sano, en dicho estudio sólo se observó un impacto significativo en los indicadores de enfermedad diarreica aguda. La falta de impacto en los resultados a nivel de las otras variables estaría relacionado a que el logro de dichos resultados dependen no sólo del financiamiento a través del Seguro Integral de Salud, sino principalmente de presupuesto transferido directamente a las regiones por "Recursos Ordinarios" desde el MEF.

El Seguro Integral de salud para su implementación del mecanismo capitado, no previó el desarrollo de una evaluación prospectiva bajo metodología experimental de un grupo de intervención y de un grupo control, por lo que la decisión de escalar el mecanismo de pago hacia el año 2013 para todo el país, se debió principalmente a la observación del incremento de las prestaciones preventivas en las regiones del ámbito cápita durante el año 2012, asumiendo que dicho incremento se debía a la intervención.

Dicha premisa por parte del Seguro Integral de Salud, no necesariamente era cierta, por cuanto se asumió bajo una evaluación pre-post; que la situación de dichas regiones iba a continuar exactamente igual para el año 2012 respecto del año 2011.

Los diseños simples pretest-postest. Son quizás los más conocidos y constituyen una alternativa viable cuando pueden ser obtenidas medidas de las variables relevantes con carácter previo a la intervención. Sin embargo, desde el punto de vista de la validez interna, aporta pocas garantías. La diferencia entre las medidas pre y post pueden ser debidas a factores ajenos a la propia intervención y este tipo de diseños no permite deslindar el efecto neto del programa ${ }^{14}$.

Por lo tanto, si bien existían datos que razonablemente llevaban a la conclusión de que la intervención estaba generando el incremento de la producción de atenciones preventivas en las regiones, la validez interna de esta conclusión no estaba garantizada, asimismo el escalar a nivel país en el año 2013 no permitió contar con un grupo de regiones que se encuentren fuera del mecanismo capitado durante más tiempo.

En este contexto es que se opta por la elección de esta metodología para explicar el incremento de las prestaciones preventivas ocurridas desde el año 2013 hasta el año 2016 desde el mecanismo capitado.

Al respecto existe la metodología de evaluación de "Diferencia de Diferencias" que tiene claras ventajas para la evaluación, controla por todas las características que no cambian con el tiempo (tanto observables como no observables) y por todos los cambios en el tiempo que afectan al grupo tratado y no tratado de igual manera.

Para mayor validez aún se eligió un grupo de regiones del contrafactual apareadas por un Indicador el Índice de Desarrollo Humano (IDH).

Por lo tanto los resultados obtenidos tienen una validez interna significativa y podrían ser utilizados para explicar la razón por la cual se lograron los mismos desde una relación de causalidad.

El efecto positivo del mecanismo capitado en el Seguro Integral de Salud también había sido ya observado en años anteriores. El primer logro claramente observado luego de la implementación, fue la inversión en la frecuencia de prestaciones preventivas frente a las recuperativas así como el mantenimiento de la dirección del ratio preventivo/recuperativo. Estos resultados representan un cambio de conducta en el prestador que orienta mayores esfuerzos en relación a la prevención y deja de considerar a la prestación como el centro, para buscar el resultado sanitario que se logra con una buena gestión del financiamiento ${ }^{18}$.

Debido al peso específico del financiamiento del Seguro Integral de Salud, no resulta pertinente, tratar de explicar comportamientos a nivel de indicadores sanitarios finales, debido a que dichas metas se financian mayoritariamente desde "Recursos Ordinarios" por el Ministerio de Economía y Finanzas.

\section{CONCLUSIÓN}

La ejecución del presupuesto a las regiones, por la fuente de financiamiento Donaciones y Transferencias 
desde el Seguro Integral de Salud, se incrementó del $68,5 \%$ en el 2012 al 92,3\% el 2016.

En relación a las prestaciones preventivas, en base a los resultados del 2013, se observa un incremento en las prestaciones preventivas; también sucede lo mismo con la inversión de la relación preventiva/recuperativa manteniéndose la tendencia en los siguientes años evaluados.

Las regiones seleccionadas como piloto cápita, durante el año 2012, lograron una diferencia de 1551 prestaciones preventivas, por cada diez mil asegurados, versus regiones que no estuvieron incluidas en dicho piloto.

De lo observado, se puede indicar que el mecanismo de pago capitado por el SIS se confirma tres resultados esperados: i) Incremento del porcentaje de ejecución en las regiones de los recursos transferidos por la fuente "Donaciones y Transferencias", ii) Inversión de la relación preventiva/recuperativa en favor de las prestaciones preventivas $y$, iii) Resultados de incremento de prestaciones preventivas superiores en las regiones piloto versus el Contrafactual.
Esta información es puesta a disposición para ser tomada en cuenta por las diferentes IAFAS públicas y en especial por el Seguro Integral de Salud para la toma de decisiones respecto a la decisión de retomar el mecanismo capitado desarrollado durante los años 2012 al 2016.

Contribuciones de autoría: Los autores participaron la génesis de la idea, diseño de proyecto, recolección e interpretación de datos, análisis de resultados y preparación del manuscrito del presente trabajo de investigación.

Financiamiento: Autofinanciado.

Conflicto de interés: Los autores declaran no tener conflicto de interés en la publicación de este artículo.

Recibido: 20 de marzo 2019

Aprobado: 20 de mayo 2019

Correspondencia: Pedro Grillo-Rojas. Evaluación del mecanismo de pago capitado en el seguro integral de salud del Perú.

Dirección: Alameda del Corregidor 1502, La Molina 15024

Correo:drgrillorojas@yahoo.com

\section{REFERENCIAS BIBLIOGRÁFICAS}

1. Arriaga P, Valdez M. Acuerdo Nacional .31 Políticas de estado del acuerdo nacional del Perú. PNUD: Perú;2014.

2. Ministerio de Salud. Ley $N^{\circ} 29344$ y aseguramiento universal del Perú. MINSA: Lima; 2018.

3. Ministerio de Salud. Ley $N^{\circ} 29344$ y aseguramiento universal del Perú MINSA: Lima; 2011.

4. Castaño RA. Mecanismos de pago en Salud, Anatomía, Fisiología y Fisiopatología. 2da Ed. Ediciones Ltda: Colombia; 2000.

5. Organización Mundial de Salud. Informe sobre la salud en el mundo. El camino hacia la cobertura universal. Ginebra; 2010.

6. Ministerio de Salud. Guía Técnica para el Cálculo del Cápita. MINSA Perú; 2016

7. Ministerio de Salud. Guías Técnicas para las Transferencias Financieras en el Primer Nivel de Atención. MINSA: Perú; 2016.

8. Congreso de la República. Comisión de defensa nacional, orden interno, desarrollo alternativo, y lucha contra las drogas. Perú; 2017.

9. Raña K Javier, Ferrer O Juan-Carlos, Bedregal G Paula. Modelo de asignación de recursos en atención primaria. Rev. méd. Chile . 2007. 135(1): 54-62.
10. Informe Regional del Desarrollo Humano. Seguridad ciudadana con rostro humano: diagnóstico y propuestas para América Latlna. PNUD: Panamá; 2013.

11. Unidad Funcional de Estadística. Boletín estadístico mensual. MINSA: LIMA; 2019.

12. Villacrés T, Mena AC. Mecanismos de pago y gestión de recursos financieros para la consolidación del Sistema de Salud de Ecuador. Rev Panam Salud Publica. 2017;41:51.

13. Roberto Romero-Onofre, et Al.. Impacto prestacional y sanitario de financiamiento capitado en gestantes y niños menores de 5 años en Perú, 2011 - 2014. RFMH. 2018;18(3):14-19.

14. Apodaca PM. Evaluación de los resultados y del impacto. Rev Inv Edu. 1999; 12(2): $363-377$.

15. Pomeranz D. Métodos de Evaluación. Harvard Business School . 2011; $10(2): 1-12$.

16. Tejerina L, Bouillon C, Demaestri E.. Financial services and proverty reduction in Latin America and the Caribbean. Inter-American Development Bank.: Washintong D.C.; 2006. 